\title{
Approximation Theory for Matrices
}

\author{
A. D. Kennedy ${ }^{\mathrm{a}}$ \\ a School of Physics, University of Edinburgh, King's Buildings, Edinburgh, EH9 3JZ, United Kingdom \\ We review the theory of optimal polynomial and rational Chebyshev approximations, and Zolotarev's formula \\ for the sign function over the range $\epsilon \leq|z| \leq 1$. We explain how rational approximations can be applied to \\ large sparse matrices efficiently by making use of partial fraction expansions and multi-shift Krylov space solvers.
}

\section{Introduction}

There are many situations in which it is desirable to evaluate a function of a matrix. For instance, in lattice quantum field theory it is sometimes desirable to evaluate the square root of a discretised Dirac operator $\not D$ in order to calculate the effects of varying the number of fermionic flavours [1-5], or to construct a good approximation to Neuberger's operator for Ginsparg-Wilson fermions by evaluating the sign function $[6-9]$.

\section{Matrix Functions}

As a general mathematical problem we need to define what we mean by the generalisation of a scalar function to a corresponding matrix-valued function on matrices. This can be done in a variety of ways [10], but for our purposes it suffices to restrict our attention to the special case of an Hermitian matrix $H$, which can be transformed into real diagonal form by a unitary transformation ${ }^{1} H=U D U^{\dagger}$. Defining a function of a real diagonal matrix to be a diagonal matrix in the obvious way, $f(D)_{i i} \equiv f\left(D_{i i}\right)$, we may define $f(H) \equiv U f(D) U^{\dagger}$.

We next address the question of how to compute good numerical approximations to $f(H)$ cheaply for a useful class of functions. One way of doing this is to find a polynomial or rational function which is a good approximation over a compact interval $R$ containing the spectrum of $H$. This seems a promising approach because it

\footnotetext{
${ }^{1}$ Although the lattice Dirac operator $\not D$ is not hermitian the assumption of $\gamma_{5}$ hermiticity implies that $\not D \gamma_{5}$ is, and this may be used instead.
}

is well known that rational approximations are an effective way of computing scalar functions on a digital computer [11-14].

The reason why matrix polynomials are cheap to evaluate is that they do not require explicit diagonalisation of the matrix. From the identities

$$
\begin{aligned}
U\left(\alpha D+\beta D^{\prime}\right) U^{\dagger} & =\alpha U D U^{\dagger}+\beta U D^{\prime} U^{\dagger}, \\
U D D^{\prime} U^{\dagger} & =U D U^{\dagger} U D^{\prime} U^{\dagger},
\end{aligned}
$$

we see that $p(H)=p\left(U D U^{\dagger}\right)=U p(D) U^{\dagger}$ for any polynomial $p$. Similarly, if we observe that $U D^{-1} U^{\dagger}=\left[U D U^{\dagger}\right]^{-1}$ then we see that $r(H)=$ $r\left(U D U^{\dagger}\right)=U r(D) U^{\dagger}$ for any rational function $r$, which is cheap to evaluate if we count matrix inversion as a "cheap" operation.

It is reasonable to ask why we should consider matrix inversion as "cheap." There are three practical reasons for this

1. In applications we do not need a function of a matrix or the inverse of a matrix per se, but only the effect of applying it to some vector. Therefore we only need to solve a system of linear equations, which is far cheaper than finding the full inverse.

2. There are very efficient methods for solving large systems of sparse linear equations. We may take advantage of all the work that has been done in developing and optimising such Krylov space algorithms.

3 . We can expand a rational function in partial fractions $[7,8,15]$, and then take advantage of the structure of Krylov space solvers to compute all the terms in the partial fraction expansion using the same Krylov space 
$[16,17]$. For this to work it is necessary that the partial fraction expansion is numerically stable, i.e., the terms do not suffer large cancellations; and that the denominator of the rational function is square-free. For reasons that are not fully understood both of these conditions seem to be satisfied in practice.

Observe that the definition of $f(H)$ only requires that we know the values that $f$ takes on the eigenvalues of $H$, whereas the polynomial or rational approximation must be valid over an interval containing the spectrum. For certain functions, such as $1 / x$, we can find cheaper methods: for instance, Krylov space methods are usually much better for finding an inverse than using a polynomial approximation to $1 / x$ [18].

\section{Approximation by Polynomials}

\subsection{Function Norms}

In order to define more precisely what we mean by a "good" approximation $p$ to a continuous function $f$ over the unit interval $[0,1]$ it is useful to introduce a family of norms,

$\|f\|_{n} \equiv\left(\int_{0}^{1} d x w(x)|f(x)|^{n}\right)^{1 / n}$,

where $w$ is a positive weight function and $n \geq 1$. We may verify that this does indeed define a norm on the space of continuous functions over the unit interval by the use of the Cauchy-Schwarz inequality. Some cases of especial interest are when $w(x)=1$ (absolute norm), $w(x)=1 /|f(x)|$ (relative norm), $n=2$ (Euclidean norm), and $n=\infty$ (Chebyshev norm) for which

$\|f\|_{\infty}=\max _{0 \leq x \leq 1} w(x)|f(x)|$.

In this last case if we choose the function $p$ from some special class of continuous functions, like polynomials or rational functions, then the $p$ which minimises the Chebyshev norm of $p-f$ is called the minimax approximation,

$\|p-f\|_{\infty}=\min _{p} \max _{0 \leq x \leq 1} w(x)|p(x)-f(x)|$.

If we are using floating-point numbers to represent components of spinor fields on a com- puter then it is most consistent to use a minimax approximation with a relative weight for matrix function evaluation. In particular, using an approximation with a minimax error of one $u l p$ (unit of least precision) means that the errors caused by function approximation have no reason to be worse than those we already accept by using floating-point arithmetic.

\subsection{Weierstrass' theorem}

The fundamental theorem on the approximation of continuous functions by polynomials is due to Weierstrass, who proved that any continuous function can be arbitrarily well approximated over the unit interval by a polynomial. This theorem is of significance in functional analysis, as it shows that the polynomials are dense in the space of continuous functions with respect to the topology induced by the Chebyshev norm. An elegant proof of Weierstrass' theorem may be obtained by the use of Bernstein Polynomials,

$p_{n}(x) \equiv \sum_{k=0}^{n} f\left(\frac{k}{n}\right)\left(\begin{array}{l}n \\ k\end{array}\right) x^{n}(1-x)^{n-k}$.

It is simple to show that $\lim _{n \rightarrow \infty}\left\|p_{n}-f\right\|_{\infty}=0$.

\section{Optimal Approximation}

Weierstrass' theorem tells us that for any specified tolerance $\epsilon>0$ we can find a degree $n$ such that there is a polynomial $p_{n}$ satisfying $\left\|p_{n}-f\right\|_{\infty}<\epsilon$. For our purposes the converse question is of greater importance, namely for a given degree $n$ what is the smallest error $\left\|p_{n}-f\right\|_{\infty}$ that can be achieved. This question was addressed by Chebyshev, who provided a suprisingly simple answer. For pedagogical reasons we shall first discuss Chebyshev's theorem for the case of approximation by polynomials, and then generalise the result to rational functions (ratios of polynomials); although logically the polynomial case is just a special case of the rational one where the degree of the denominator polynomial happens to be zero. 


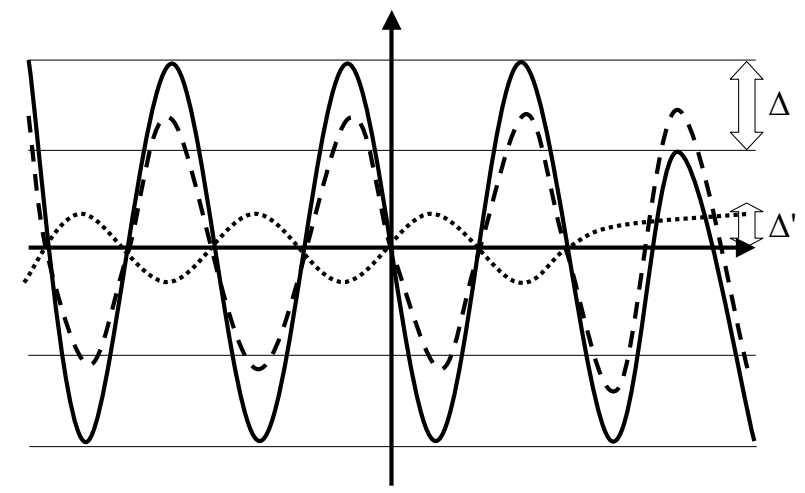

Figure 1. The solid line shows the error $e$ for a polynomial approximation $p$ of degree $d$ to the continuous function $f$, which has less than $d+2$ extrema of equal magnitude. This defines a "gap" $\Delta$ between the extrema. We may construct the polynomial $q$ of degree $d$ shown by the dotted line that has opposite sign to the error at each of the extrema, and whose magnitude $\Delta^{\prime}<\Delta$. The polynomial $p+q$ is then a better approximation than $p$, as is obvious from its error $w(x)[p(x)+$ $q(x)-f(x)]$ shown by the dashed line.

\subsection{Polynomials}

Chebyshev proved that for any degree $d$ there is always a unique polynomial ${ }^{2} p$ that minimises $\|e\|_{\infty}=\max _{0 \leq x \leq 1}|e(x)|$, where the error is $e(x) \equiv w(x)[p(x)-f(x)]$. Furthermore this polynomial is characterised by the criterion that the error $e(x)$ takes its maximum absolute value at at least $d+2$ points on the unit interval (which may include the end points of the interval), and the sign of the error alternates between its successive extrema.

The proof of Chebyshev's theorem is straightforward, and is illustrated in Figures 1 and 2. First we prove that Chebyshev's criterion is necessary: if the error attains its extreme value at fewer

\footnotetext{
2The polynomial may be of degree less than $d$; for example the best approximation of any degree to a constant is just that constant. A less trivial example is that the zero polynomial is the best approximation to $\sin N \pi x$ over $[0,1], N \in \mathbb{N}$, for all $d \leq N$.
}

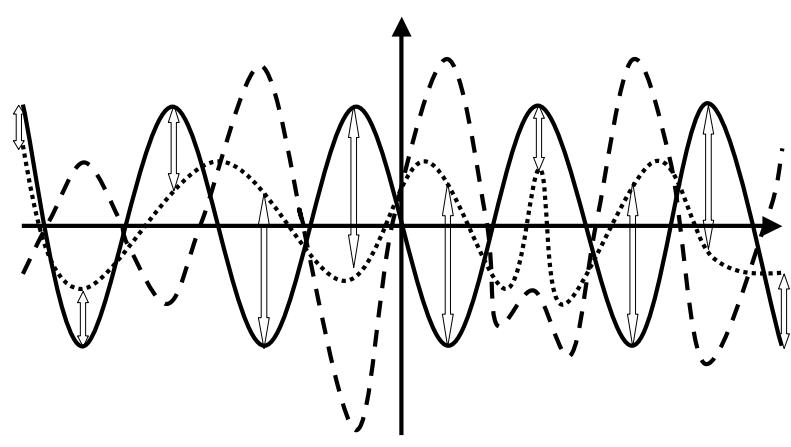

Figure 2. The solid curve is the error $e_{p}$ for a polynomial $p$ of degree $d$ satisfying Chebyshev's criterion. The dotted line shows the error $e_{p^{\prime}}$ for a hypothetical better approximation $p^{\prime}$. The dashed line is the polynomial $p^{\prime}-p$ which must have at least $d+1$ zeros and therefore vanishes identically.

than $d+2$ "alternating" points then the approximation can be improved. Consider a polynomial $p$ for which the error $e(x) \equiv w(x)[p(x)-f(x)]$ takes its extreme values at fewer than $d+2$ points (shown by the solid curve in Figure 1); the next largest extremum of the error (which may be a local extremum or may occur on the boundary) must be smaller by some non-zero "gap" $\Delta$. As the $d+1$ extrema alternate in sign the error must pass through zero between each successive extremum, and there are at most $d$ such zeros $z_{i}$ (if there are several zeros between adjacent extrema we just chose one of them). We can construct a polynomial $u$ of degree $d$ (shown by the dotted line in the figure) which is zero at each of the $z_{i}$. This may be written explicitly as $u(x) \equiv A \prod_{i}\left(x-z_{i}\right)$. The constant $A$ may be chosen such that the sign of $u$ is opposite to that of the error at each extremuum, and its magnitude $\Delta^{\prime}=\|u\|_{\infty}=\max _{0 \leq x \leq 1} w(x)|u(x)|$ is less than the gap $\Delta$. It follows that the polynomial $p+u$ is a better approximation to $f$, as its error $p(x)+u(x)-f(x)$ (the dashed line in the figure) is everywhere less than $e(x)$.

Next we prove the sufficiency of Chebyshev's 
criterion: if the error attains its extreme values at exactly $d+2$ alternating points then it is indeed the optimal approximation. To show this assume that there was a polynomial $p^{\prime}$ that furnished a better approximation. The solid line in Figure 2 shows the error $e_{p}(x) \equiv$ $w(x)[p(x)-f(x)]$ for a polynomial $p$ satisfying Chebyshev's criterion. The dotted line shows the error $e_{p^{\prime}}(x) \equiv w(x)\left[p^{\prime}(x)-f(x)\right]$ for the hypothetical better approximation $p^{\prime}$. Since $p^{\prime}$ is a better approximation $\left\|e_{p^{\prime}}\right\|_{\infty}<\left\|e_{p}\right\|_{\infty}$, or equivalently $\left|e_{p^{\prime}}\left(x_{i}\right)\right|<\left|e_{p}\left(x_{i}\right)\right|$ at each of the $d+2$ extrema $x_{i}$ of $e_{p}(x)$. Appealing to continuity we deduce that $e_{p^{\prime}}\left(z_{i}\right)=e_{p}\left(z_{i}\right)$ at at least $d+1$ points $z_{i}$ between the extrema. From this it follows that $e_{p^{\prime}}\left(z_{i}\right)-e_{p}\left(z_{i}\right)=w\left(z_{i}\right)\left[p^{\prime}\left(z_{i}\right)-f\left(z_{i}\right)\right]-$ $w\left(z_{i}\right)\left[p\left(z_{i}\right)-f\left(z_{i}\right)\right]=w\left(z_{i}\right)\left[p^{\prime}\left(z_{i}\right)-p\left(z_{i}\right)\right]=0$, so the polynomial $p^{\prime}(x)-p(x)$ (shown by the dashed line) must have a least $d+1$ zeros, but as it is of degree $d$ this means it is identically zero by the fundamental theorem of arithmetic. Hence $p^{\prime}=p$ and the polynomial $p$ is both optimal and unique.

\subsection{Chebyshev Polynomials}

The Chebyshev polynomials ${ }^{3} T_{n}(\cos \theta) \equiv$ $\cos n \theta$ provide a simple example of optimal minimax approximations. $T_{n}(x)$ obviously has $n+1$ extrema which alternate in value between -1 and 1 for $-1 \leq x \leq 1$; therefore $p_{n}(x) \equiv$ $x^{n}-2^{1-n} T_{n}(x)$ is the best polynomial approximation of degree $n-1$ with uniform weight to the function $x^{n}$ over the interval $[-1,1]$, for the error $e_{n}(x) \equiv p_{n}(x)-x^{n}=2^{1-n} T_{n}(x)$ satisfies Chebyshev's criterion. The magnitude of the error is just $\left\|e_{n}\right\|_{\infty}=2^{1-n}=2 e^{-n \ln 2}$, so we see that in this case the error decreases exponentially with respect to $n$.

If we consider a function $f$ that has a conver-

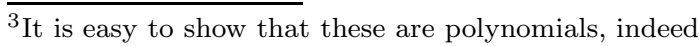

$T_{n}(x)=\sum_{k=0}^{\lfloor n / 2\rfloor}\left(\begin{array}{c}n \\ 2 k\end{array}\right) \sum_{\ell=0}^{k}\left(\begin{array}{c}k \\ \ell\end{array}\right)(-1)^{\ell} x^{n-2 \ell}$.

The leading coefficient of $T_{n}(x)$, that is the coefficient of $x^{n}$, is thus seen to be $\sum_{k=0}^{\lfloor n / 2\rfloor}\left(\begin{array}{c}n \\ 2 k\end{array}\right)=2^{n-1}$. gent Taylor series expansion over $[-1,1]$,

$f(x)=\sum_{j=0}^{\infty} \frac{f^{(j)}(0)}{j !} x^{j}$,

and we reexpress it as an expansion in Chebyshev polynomials $^{4}$

$f(x)=\sum_{j=0}^{\infty} c_{j} T_{j}(x)$

then truncating this expansion at order $d$ gives an approximation close to the optimal one of this degree. It is in fact the optimal polynomial approximation to the truncated Taylor expansion of degree $d+1$, which is not necessarily exactly the optimal one. Likewise, the convergence of the truncated Chebyshev expansion may converge exponentially in $d$, but it will not if the original Taylor series expansion did not converge exponentially.

\subsection{Rational Functions}

It is perhaps surprising that Chebyshev's argument can be easily extended to the case of optimal rational approximations as well. The statement of Chebyshev's theorem in this case is that for any degree $(n, d)$ there is always a unique rational function $r$ that minimises $\|e\|_{\infty}=\max _{0 \leq x \leq 1}|e(x)|$, where the error is $e(x) \equiv w(x)[r(x)-f(x)]$. For rational approximations Chebyshev's crtierion is that the error $e(x)$ takes its maximum absolute value at at least $n+d+2$ points on the unit interval (which may include the end points of the interval), and the sign of the error alternates between its successive extrema.

The proof is similar to that for polynomials given above, and we shall therefore just briefly consider the salient differences. To prove that Chebyshev's criterion is necessary we consider a degree $(n, d)$ rational function $r=p / q$ for which

${ }^{4}$ This is straightforward because they satisfy the orthogonality relation

$\frac{2}{\pi} \int_{-1}^{1} \frac{d x}{\sqrt{1-x^{2}}} T_{n}(x) T_{m}(x)=\delta_{n, m}$

(apart from the trivial case where $n=m=0$ for which there is an additional factor of two). 
the error $e \equiv r-f$ attains its extreme value $\|e\|_{\infty}$ at fewer than $n+d+2$ alternating points. The denominator $q$ cannot have any zeros on the unit interval, as clearly $q=1$ would then lead to a smaller error; therefore without loss of generality we can assume $q(x)>\delta>0$ throughout the interval. Just as before we may construct the polynomial $u$ of degree $n+d$ that shares the zeros of the $e$. Since $\operatorname{gcd}(p, q) \neq 0$ we can construct polynomials $s$ and $t$ of degrees $d$ and $n$ such that $u=s p+t q$ using the extended Euclidean algorithm. We then define the rational function $r^{\prime} \equiv \frac{p-\epsilon t}{q+\epsilon s}$ which satisfies

$$
\begin{aligned}
\left|r^{\prime}-f\right| & =\left|\frac{p-\epsilon t}{q+\epsilon s}-\frac{p}{q}+\frac{p}{q}-f\right| \\
= & \left|-\frac{\epsilon(q t+p s)}{q(q+\epsilon s)}+e\right|=\left|e-\frac{\epsilon u}{q(q+\epsilon s)}\right|
\end{aligned}
$$

Choosing the constant $\epsilon$ small enough that $q(x)+$ $\epsilon s(x)>\delta^{\prime}>0$ for $x \in[0,1]$ and $\epsilon\|u\|_{\infty}<\Delta \delta \delta^{\prime}$ the required result follows.

The proof of sufficiency in the rational case is even simpler. If $r=p / q$ is a degree $(n, d)$ rational approximation whose error $w(r-f)$ has $n+d+2$ alternating extrema of equal magnitude, and $r^{\prime}=p^{\prime} / q^{\prime}$ is a hypothetical better rational approximation of the same degree, then the error for $r^{\prime}$ must cross that for $r$ at least $n+d+1$ times, i.e., the rational function $r-r^{\prime}$ must have more than $n+d$ zeros. Now, in order for

$r-r^{\prime}=\frac{p}{q}-\frac{p^{\prime}}{q^{\prime}}=\frac{p q^{\prime}-p^{\prime} q}{q q^{\prime}}$

to vanish on the unit interval the numerator must vanish (the denominator certainly cannot); and the polynomial $p q^{\prime}-p^{\prime} q$ is of degree $n+d$ and thus can only have $n+d+1$ zeros if it vanishes identically, hence $r=r^{\prime}$.

\section{Remez Algorithm}

Chebyshev's theorem assures us that there is a unique ${ }^{5}$ optimal rational approximation of any degree to any continuous function over the unit interval, and it even provides a simple criterion for the behaviour of the error in this case, which

\footnotetext{
${ }^{5}$ In some cases the optimal approximation may actually
} be of lower degree. is certainly of practical use in verifying that a putative minimax solution is correct. In fact, the proof of the theorem is quite constructive, but it does not provide a particularly efficient means of computing the optimal solution in practice.

In general, the computation of the optimal Chebyshev rational approximation is often performed using the Remez algorithm [19,14]. To describe the Remez algorithm we define a reference $X$ as a set of points $\left\{x_{i} \in[0,1], i=\right.$ $1, \ldots, n+d+2\}$ that we will make converge to the alternating set at which the error takes its extreme values. The Remez algorithm alternates two steps:

1. New $r$. Keep $X$ fixed, and choose the best rational approximation $r$ which passes through the points $\left(x_{i}, f\left(x_{i}\right)+(-1)^{i} \Delta\right)$ for $x_{i} \in X$.

2. New $X$. Keep $r$ fixed, and choose a new reference which is the best alternating set for $r$ near to $X$.

In slightly more detail:

1. Interpolate an $(n, d)$ rational function $r=p / q$ such that the error $e\left(x_{i}\right) \equiv$ $w\left(x_{i}\right)\left[f\left(x_{i}\right)-r\left(x_{i}\right)\right]=(-1)^{i} \Delta$. Setting $p(x) \equiv \sum_{j=0}^{n} p_{j} x^{j}$ and $q(x) \equiv \sum_{j=0}^{d} q_{j} x^{j}$ with $q_{d}=1$ this gives the $n+d+2$ equations $\sum_{j=0}^{d} q_{j} x_{i}^{j}\left[f\left(x_{i}\right)-(-1)^{i} \Delta / w\left(x_{i}\right)\right]-$ $\sum_{j=0}^{n} p_{j} x_{i}^{j}=0$ for the $n+d+2$ variables $p_{j}, q_{j}$, and $\Delta$. Noting that these equations are linear in the $p_{j}$ and $q_{j}$, we write this as the matrix equation $M v=0$ where $v$ is the vector $\left(p_{0}, \ldots, p_{n}, q_{0}, \ldots, q_{n}\right)$. This has a non-trivial solution for $v$ iff $\operatorname{det} M=0$, which is a polynomial in $\Delta$. For the values of $\Delta$ which are real roots of this polynomial we can find the coefficients $p_{j}$ and $q_{j}$, and check that the resulting rational function $r$ is valid.

2. Choose a partition of $[0,1]$ into intervals $I_{i}$ such that $x_{i} \in I_{i}$, and choose a new reference $X^{\prime} \equiv\left\{x_{i}^{\prime} \in I_{i}:(-1)^{i} e\left(x_{i}^{\prime}\right)=\right.$ $\left.\max _{x \in I_{i}}(-1)^{i} e(x)\right\}$.

The Remez algorithm has several drawbacks: 
- It can sometimes be hard to find a suitable initial reference: the location of the zeros of Chebyshev polynomials are sometimes suggested as an ad hoc guess.

- The construction of the interpolating rational function $r$ can fail for some exceptional references.

- Finding the extrema of $e(x)$ in an interval $I_{i}$ can be tricky if the function $f$ oscillates rapidly within the interval. As $f$, and therefore $e$, can be any continuous function an efficient yet robust algorithm for locating the extrema is hard to find.

- The computation often has to be carried out in multiple-precision arithmetic: it is not unusual to need 20-30 or more decimal digits precision.

- The rate of convergence is quite slow.

Of course, if bounds on the spectrum of a family of matrices is known a priori then the cost of computing the optimal rational approximation is not too significant, as it only has to be done once for many matrix function evaluations. This is the case, for example, in computing roots of the Dirac operator for staggered fermions, whose spectrum is bounded above by a constant and below by the mass. On the other hand, in the case of Wilson fermions or massless overlap GinspargWilson fermions a useful lower bound on the spectrum does not exist, and one either has to use a higher-degree approximation covering a conservative range or one has to recompute the approximation "on the fly," both of which may be prohibitively expensive options.

\section{Zolotarev's Theorem}

It is fortunate that in the most interesting cases, namely square roots, inverse square roots, and the sign function $\operatorname{sgn}(x)$ the coefficients of the optimal Chebyshev rational approximation are known analytically. This result is due to Zolotarev, who was a student of Chebyshev.

Zolotarev [20] gave an explicit expression for optimal rational approximations to the sign function. To be precise, he gave an expression of the

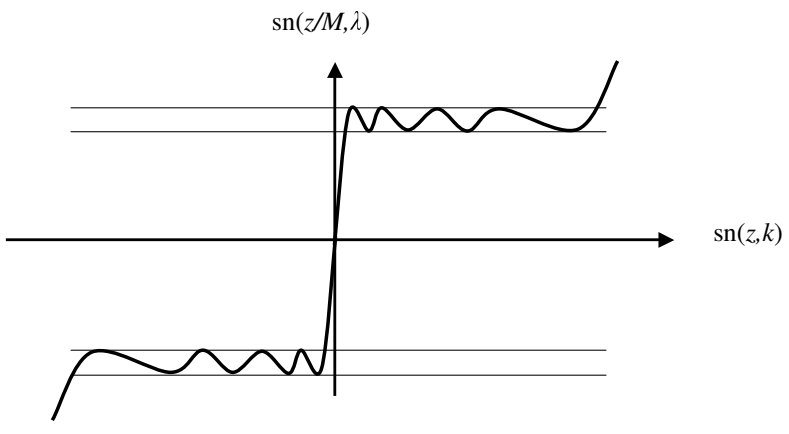

Figure 3. Sketch of the function $r(x)$ of equations (1) and (2). The four values $\pm 1 \pm \Delta$ are indicated by the horizontal lines, and $r(x)$ crosses these lines at $\pm \epsilon$ and \pm 1 .

form $r(x)=x R\left(x^{2}\right)$ where $R$ is a rational function and

$\max _{\epsilon \leq|x| \leq 1}|r(x)-\operatorname{sgn}(x)|=\Delta$.

Note that this degree $(n+1, n)$ approximation is as near to a "diagonal" rational function as possible, since $r$ must be odd. Furthermore, no approximation with lower degree numerator could be better, for otherwise it would violate the uniqueness part of Chebyshev's theorem.

The approximation is illustrated in Figure 3. It is clear that $\tilde{r}(x)=\left(1-\Delta^{2}\right) / r(x)$ is an optimal approximation to $\operatorname{sgn}(x)$ of degree $(n, n+1)$, which is singular rather than zero at $x=0$. If there was a better approximation than $r$ with higher degree numerator then $\tilde{r}$ would not be unique, again contrary to Chebyshev's theorem.

\subsection{Zolotarev Coefficients}

It is amusing that whereas the properties of Chebyshev polynomials follow from the fact that $\cos n \theta$ is a polynomial in $\cos \theta$, Zolotarev's theorem follows from an analogous result for elliptic functions.

The Jacobi elliptic function sn is implicitly defined as the inverse of an elliptic integral, namely

$z \equiv \int_{0}^{\operatorname{sn}(z ; k)} \frac{d t}{\sqrt{\left(1-t^{2}\right)\left(1-k^{2} t^{2}\right)}}$. 
It is a doubly periodic analytic function in the complex plane with periods $\omega=4 \mathrm{~K}(k)$ and $\omega^{\prime}=$ $2 i \mathrm{~K}\left(k^{\prime}\right)$, where $\mathrm{K}$ is the complete elliptic integral

$\mathrm{K}(k) \equiv \int_{0}^{1} \frac{d t}{\sqrt{\left(1-t^{2}\right)\left(1-k^{2} t^{2}\right)}}$

and $k^{2}+k^{\prime 2}=1$. It can be shown $[21,22]$ that any elliptic function with these periods must be expressible as a rational function of $\operatorname{sn}(z ; k)$ and the related Jacobi elliptic functions $\operatorname{cn}(z ; k)=$ $\sqrt{1-\operatorname{sn}(z ; k)^{2}}$ and $\operatorname{dn}(z ; k)=\sqrt{1-k^{2} \operatorname{sn}(z ; k)^{2}} ;$ and more specifically that any even elliptic function with the same periods is a rational function of $\operatorname{sn}(z ; k)^{2}$. In fact, any elliptic function with equivalent periods, in the sense that they generate the same period lattice, are rationally expressible in this manner; as are elliptic functions with periods which divide $\omega$ and $\omega^{\prime}$, since the latter are also periodic with the original periods. Such rational relationships are known as modular transformations.

Consider in particular an elliptic function with periods $\tilde{\omega}=\omega=4 \mathrm{~K}(k)$ and $\tilde{\omega}^{\prime}=\omega^{\prime} / n=$ $2 i \mathrm{~K}\left(k^{\prime}\right) / n$. Such a function is easily constructed from $\operatorname{sn}(z ; k)$ by scaling the argument $z$ by some factor $1 / M$ and choosing a new parameter $\lambda$. The function $\operatorname{sn}(z / M ; \lambda)$ has periods $4 L=4 \mathrm{~K}(\lambda)$ and $2 i L^{\prime}=2 i \mathrm{~K}\left(\lambda^{\prime}\right)$ where $\lambda^{2}+\lambda^{\prime 2}=1$, so the periods with respect to $z$ are $4 L M$ and $2 i L^{\prime} M$, which thus requires $L M=\mathrm{K}(k)$ and $L^{\prime} M=\mathrm{K}\left(k^{\prime}\right) / n$. The even elliptic function $\operatorname{sn}(z / M ; \lambda) / \operatorname{sn}(z ; k)$ must therefore be expressible as a rational function $R$ of $\operatorname{sn}(z ; k)^{2}, \operatorname{sn}(z / M ; \lambda)=\operatorname{sn}(z ; k) R\left(\operatorname{sn}(z ; k)^{2}\right)$.

Matching the poles and zeros, and fixing the overall normalisation from the behaviour near $z=0$, the coefficients of the numerator and denominator polynomials for $R$ may be expressed in terms of Jacobi elliptic functions,

$\frac{\operatorname{sn}(z / M ; \lambda)}{\operatorname{sn}(z ; k)}=\frac{1}{M} \prod_{m=1}^{\lfloor n / 2\rfloor} \frac{1-\frac{\operatorname{sn}(z ; k)^{2}}{\operatorname{sn}\left(2 i K^{\prime} m / n ; k\right)^{2}}}{1-\frac{\operatorname{sn}(z ; k)^{2}}{\operatorname{sn}\left(2 i K^{\prime}\left(m-\frac{1}{2}\right) / n ; k\right)^{2}}}$.

The quantity $M$ is determined by evaluating this identity at the half period $\mathrm{K}(k)$ where $\operatorname{sn}(\mathrm{K}(k) ; k)=\operatorname{sn}(\mathrm{K}(k) / M ; \lambda)=\operatorname{sn}(L ; \lambda)=1$. Likewise, the parameter $\lambda$ is found by evaluating the identity at $z=\mathrm{K}(k)+i \mathrm{~K}(k) / n$.

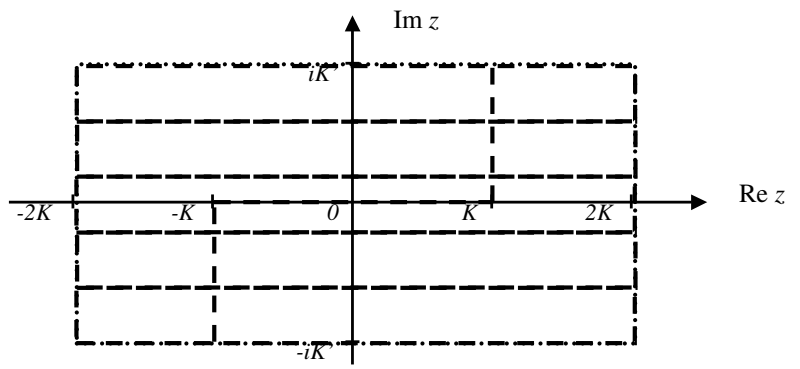

Figure 4. The fundamental region for $\operatorname{sn}(z ; k)$ is shown by the dotted box, and that for $\operatorname{sn}(z / M ; \lambda)$ whose period is divided by $n=5$ along the imaginary axis is shown by the dashed boxes. The line from $-K-i K^{\prime}$ through $-K$ and $K$ to $K+i K^{\prime}$ shows the contour in the complex $z$ plane along which both functions are real, and $\operatorname{sn}(z ; k)$ takes values from -1 through $-\epsilon$ and $\epsilon$ to 1 while $\operatorname{sn}(z / M ; \lambda)$ oscillates about it with amplitude $\Delta$.

The fundamental region for $\operatorname{sn}(z ; k)$ and $\operatorname{sn}(z / M ; \lambda)$ for $n=5$ are shown in Figure 4, which also shows the contour in the complex $z$ plane along which the modular transformation gives the desired real rational relationship between these two elliptic functions. Some further insight is given by Figure 5, in which the real part of $\operatorname{sn}(z / M ; \lambda)$ is shown over the same region of the complex plane. The imaginary part vanishes along the contour described in Figure 4, which is also indicated on this plot. From this plot it is clear how the two periods of the elliptic functions correspond to the "step" of the sign function and the oscillations of $r$ about it.

6.2. Approximations to $\sqrt{x}$ and $1 / \sqrt{x}$

We may rewrite equation (1) in the form

$$
\begin{aligned}
\Delta & =\max _{\epsilon \leq|x| \leq 1}\left|x R\left(x^{2}\right)-\frac{x}{|x|}\right| \\
& =\max _{\epsilon \leq|x| \leq 1}\left|R\left(x^{2}\right)-\frac{1}{|x|}\right| \cdot|x|
\end{aligned}
$$

and, upon setting $\xi=x^{2}$, we find the relation $\Delta=\max _{\sqrt{\epsilon} \leq \xi \leq 1}\left|R(\xi)-\frac{1}{\sqrt{\xi}}\right| \cdot \sqrt{\xi}$. This shows 


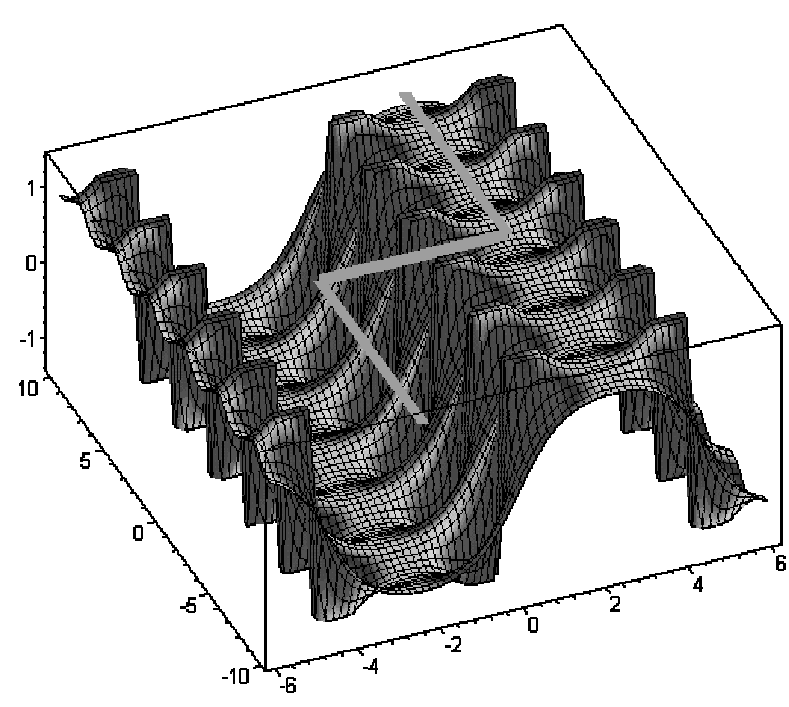

Figure 5. Surface plot showing the real part of $\operatorname{sn}(z / M ; \lambda)$ over the fundamental region of $\operatorname{sn}(z ; k)$. The imaginary part vanishes along the contour indicated.

that $R(x)$ is an optimal Chebyshev approximation to the function $1 / \sqrt{x}$ with weight $\sqrt{x}$ over the interval $[\sqrt{\epsilon}, 1]$, or in other words the optimal Chebyshev approximation with unform relative error $\Delta=\max _{\sqrt{\epsilon} \leq \xi \leq 1} \frac{|R(\xi)-1 / \sqrt{\xi}|}{1 / \sqrt{\xi}}$. We can rewrite this as $\Delta=\max _{\sqrt{\epsilon} \leq \xi \leq 1} \frac{|\xi R(\xi)-\sqrt{\xi}|}{\sqrt{\xi}}$, so $x R(x)$ is an optimal Chebyshev approximation to $\sqrt{x}$ over $[\sqrt{\epsilon}, 1]$ with uniform relative error.

\section{Examples}

\subsection{A Simple Numerical Example}

It might be useful to look at an actual example of the optimal minimax rational approximations we have been discussing. The optimal degree $(3,3)$ approximation to $1 / \sqrt{x}$ over the interval $[0.003,1]$ is

$$
\frac{1}{\sqrt{x}} \approx 0.3904603901 \frac{(x+2.3475661045)}{(x+0.4105999719)} \times
$$

$$
\times \frac{(x+0.1058344600)(x+0.0073063814)}{(x+0.0286165446)(x+0.0012779193)},
$$

and, as promised, it has a numerically stable partial fraction expansion

$$
\begin{aligned}
\frac{1}{\sqrt{x}} & \approx 0.3904603901+\frac{0.0511093775}{x+0.0012779193}+ \\
& +\frac{0.1408286237}{x+0.0286165446}+\frac{0.5964845033}{x+0.4105999719}
\end{aligned}
$$

Notice that all the terms are positive, and the roots of the denominators are all negative.

\subsection{Comparison of Polynomial and Ratio- nal Approximations}

We now briefly compare the quality of polynomial and rational approximations. It is common folklore that even though they require division operations rational approximations are superior to polynomial ones, and this seems to be true for matrix function approximations too, if use is made of the partial fraction expansion and multi-shift solver tricks. In a sense, as we mentioned before, this is because Krylov space solvers are more effective than polynomial approximations to $1 / x$ of fixed degree as a means of solving systems of linear equations.

A numerical comparison of the minimax errors for optimal rational and polynomial approximating functions to $1 / \sqrt{x}$ over the interval $[0.00003,1]$ (corresponding to a staggered fermion mass parameter of $m=0.025$ ) as a function of approximation degree is shown in Figure 6. The key points to note are:

- The error of the rational approximations falls exponentially with their degree. Machine-precision errors of one ulp (about $10^{-7}$ for 32 -bit IEEE floating-point arithmetic and $10^{-15}$ for 64 -bit arithmetic) are easily achieved for relatively low degree rational functions.

- In order to reach small errors, of the scale of the floating-point error scale of one ulp, using polynomials requires extemely high degree polynomials. Considerable care needs to be taken with regard to rounding errors in these cases. 


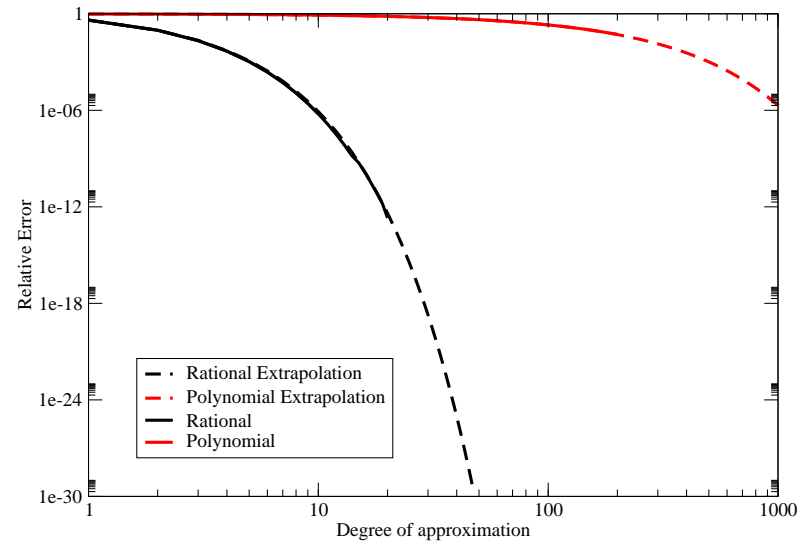

Figure 6. Comparison of minimax errors for optimal rational and polynomial approximating functions to $x^{-1 / 2}$ over the interval $[0.00003,1]$ (corresponding to a staggered fermion mass parameter $m=0.025)$ as a function of approximation degree.

- The amount of work needed to apply the rational approximations of a sparse matrix to a vector, using a partial fraction expansion and multi-shift solver, depends essentially on the degree of the rational function times the dimension of the Krylov space required.

The dependence of the minimax error $\Delta$ for the Zolotarev rational approximations on the approximation interval $[\epsilon, 1]$ and the degree $n$ is shown in Figure 7 . This plot also attempts to indicate the numerical errors in evaluating the rational function in single-precision arithmetic by the size of the squares. Again, the rapid convergence and stability of the approximations is manifest. The errors $\Delta$ appear to be consistent with an asymptotic formula of the form $\Delta \propto e^{n / \ln \epsilon}[13]$.

At the end of section 5 we noted that it was expensive to use the Remez algorithm to compute optimal rational approximations "on the fly" in the case where we do not have a useful lower bound on the spectrum of a family of Dirac operators. For the interesting cases where Zolotarev's formula is applicable we can compute the coef-

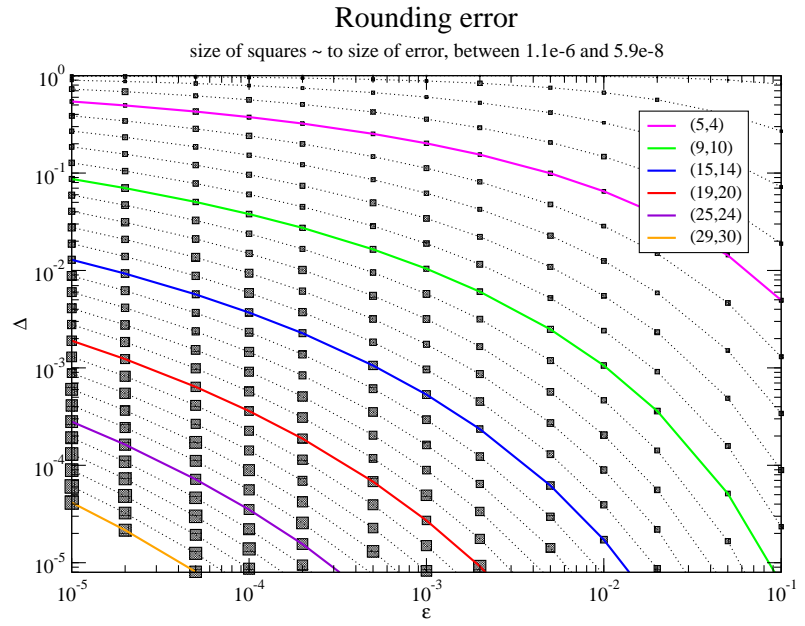

Figure 7. The minimax error $\Delta$ for Zolotarev approximations of degree $n$ over the interval $[\epsilon, 1]$. The size of the squares indicates the size of the rounding errors in evaluating the Zolotarev rational functions in single-precision arithmetic.

ficients in the rational approximation efficiently "on the fly" if we measure the smallest eigenvalue of each Dirac operator. The method of computing the Zolotarev coefficients rapidly and accurately making use of Gauss' arithmetico-geometric mean is explained in [22].

\section{Conclusion}

We reviewed the subject of minimax approximation theory, and explain why it is particularly effective for the approximation of matrix functions. We demonstrate why rational approximations are often superior to polynomial ones, and how the combination of partial fraction expansions and multi-shift Krylov space solvers allows them to be applied efficiently to large sparse matrices. Finally, we have outlined how the optimal approximation for the sign function may be found in closed form using elliptic functions. 


\section{Acknowledgments}

I would like to thank Urs Wenger and Mike Clark for help with Figures 7 and 6 respectively, and Ken Bowler for useful comments. I would also like to thank CSSM for inviting me to the very pleasant and productive workshop in Cairns.

\section{REFERENCES}

1. P. de Forcrand, Fermionic Monte Carlo algorithms for lattice QCD, (1997), hep-lat/9702009.

2. K. Jansen and R. Frezzotti, Phys. Lett. B402, 328 (1997), hep-lat/9702016.

3. I. Horváth, A. D. Kennedy, and S. Sint, A new exact method for dynamical fermion computations with non-local actions, , Nuclear Physics (Proceedings Supplements) Vol. B73, pp. 834-836, 1999, hep-lat/9809092, Proceedings of the XVIth International Symposium on Lattice Field Theory, Boulder, Colorado, 13-18 July 1998.

4. T. Takaishi and P. de Forcrand, Prepared for International Symposium on Quantum Chromodynamics (QCD) and Color Confinement (Confinement 2000), Osaka, Japan, 7-10 Mar 2000.

5. M. A. Clark and A. D. Kennedy, The RHMC algorithm for 2 flavors of dynamical staggered fermions, Nuclear Physics (Proceedings Supplements), 2003, hep-lat/0309084, Proceedings of the XXIst International Symposium Lattice Field Theory, Tsukuba, Japan, July 2003.

6. H. Neuberger, Phys. Lett. B417, 141 (1998), hep-lat/9707022.

7. H. Neuberger, Phys. Rev. Lett. 81, 4060 (1998), hep-lat/9806025.

8. R. G. Edwards, U. M. Heller, and R. Narayanan, Nucl. Phys. B540, 457 (1999), hep-lat/9807017.

9. A. Boriçi, A. D. Kennedy, B. J. Pendleton, and U. Wenger, The overlap operator as a continued fraction, , Nuclear Physics (Proceedings Supplements) Vol. B106-107, pp. 757-759, 2002, hep-lat/0110070, Proceedings of the XIXth International Symposium
Lattice Field Theory, Berlin, Germany, 19-24 August 2001.

10. G. H. Golub and C. F. V. Loan, Matrix Computations (The Johns Hopkins University Press, 1996).

11. T. J. Rivlin, An Introduction to the Approximation of Functions (Dover, 1969).

12. N. I. Achieser, Theory of Approximation (Dover, 2004).

13. P. P. Petrushev and V. A. Popov, Rational Approximation of Real Functions, Encyclopedia of Mathematics and its Applications Vol. 28 (Cambridge University Press, 1987).

14. E. W. Cheney, Introduction to Approximation Theory, 2nd ed. (American Mathematical Society, 2000).

15. T.-W. Chiu and T.-H. Hsieh, (2003), hep-lat/0306025.

16. A. Frommer, B. Nockel, S. Gusken, T. Lippert, and K. Schilling, Int. J. Mod. Phys. C6, 627 (1995), hep-lat/9504020.

17. B. Jegerlehner, (1996), hep-lat/9612014.

18. M. Lüscher, Nucl. Phys. B418, 637 (1994), hep-lat/9311007.

19. E. Y. Remez, General Computational Methods of Chebyshev Approximation (US Atomic Energy Commission, 1962).

20. E. I. Zolotarev, Zap. Imp. Akad. Nauk St. Petersburg 30, 5 (1877).

21. N. I. Akhiezer, Elements of the Theory of Elliptic Functions Vol. 79 (AMS, 1990).

22. A. D. Kennedy, Fast evaluation of Zolotarev coefficients, hep-lat/0402038, Proceedings of the Third International Workshop on $\mathrm{Nu}$ merical Analysis and Lattice QCD, NeSC, Edinburgh, June 2003. 